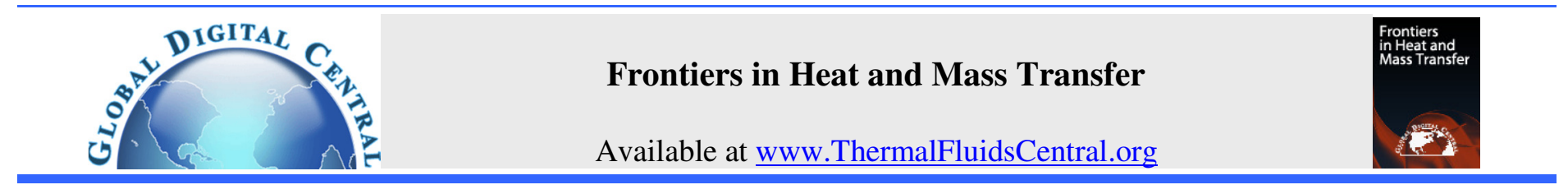

\title{
EFFECT OF NONLINEAR THERMAL RADIATION ON MAGNETOHYDRODYNAMIC WALL JET FLOW
}

\author{
M. Sathish Kumar, N. Sandeep*, B. Rushi Kumar \\ Department of Mathematics, VIT University, Vellore, 632014, India
}

\begin{abstract}
An analysis is presented to analyze the momentum and heat transfer behaviour of the laminar wall jet flow of a hydromagnetic flow due to plate with nonlinear thermal radiation and convective boundary condition. The governing Partial differential equations are converted as ordinary differential equations with the aid of similarity transformation. Further, the transformed equation is resolved using the bvc $5 \mathrm{c}$ Matlab package. The effect of various pertinent parameters on momentum and temperature fields along with the local Nusselt number is discussed with the help of numerical and graphical illustrations. It is found that the Biotnumbereffectively enhances the heat transfer rate.
\end{abstract}

Keywords: MHD, wall jet flow, thermal radiation and free convection.

\section{INTRODUCTION}

Amid the previous couple of decades, the MHD convective heat transfer flow play an important role in mechanical and engineering fields. For example, expulsion of plastic, paper drying, hot rolling, welding and so on. It gives the idea that Sakiadis (1961) initially examined the flow due to a semi-boundless evenly moving in an ambient fluid. The impact of heat transfer on steady 2-D stagnation point of Newtonian fluid were discussed by Mahapatra and Gupta $(2002,2004)$. The thermal radiation also play an important role in the heat transfer, when the coefficient of heat transfer are small, it is considered by natural convection. The impact of thermal radiation on heat transfer MHD flow past a vertical surface in dripping porous media was discussed by Bakier (2001). Mahapatra et al. (2012) studied the influence of heat generation and thermal radiation on free convection with Darcy- Forchheimer soaked porous medium and found that raising the value of thermal radiation enhance the velocity profile.

Heat transfer analysis, influenced by thermal radiation has has applications in several technological processes, including gas turbines, nuclear power plants, and the various propulsion devices for missiles aircraft, space vehicles and satellites.In radiation handle the electromagnetic waves are in charge of exchange of vitality which divert vitality from the discharging object. Pal (2013) examined the radiative stream and heat exchange over a porous extending sheet with hall current. The flow of nanofluid with blended convection and nonlinear thermal radiation were studied by Hayat et al. (2016). Rashidi et al. (2014) worked on the flow due to permeable wedge in the presence of thermal radiation and mixed convection. The effect of magnetic field and radiation on unsteady MHD flow over a vertical plate was analysed by Sandeep et al. (2014). Raju et al. (2015) worked on the MHD flow of ferrofluids past a flat plate in the presence of thermal radiation and magnetic field and found that increasing values of inclined angle increases the skin friction coefficient.

The effect of thermophoresis and Brownian motion on free convective nanofluid flow between parallel plates were investigated by Sheikholeslami and Ganji $(2016,2015,2014)$ and Sheikholeslami et al. $(2016,2016,2015)$. Vajravelu et al. (2013) discussed the unsteady MHD flow on convective heat transfer past a vertical surface with properties of variable fluid and concluded that raising value of unsteady parameter depreciate the velocity profile. The analytical solution of unsteady MHD flow caused by an incautious extending sheet was presented by Kousar and Liao (2011). Shaw et al. (2016) presented the thermal radiation effect on convection flow in the Casson fluid past a parallel plate with Soret and Dufour. They found that increasing value of velocity ratio depreciate the velocity profile. The MHD heat and mass transfer on nanofluids towards a stretching flat plate with viscous dissipation and thermal radiation was analysed by Motsumi and Makinde (2012).

Malvandi (2016) studied the magnetic field can tune the physical properties of attractive nanofluids on heat transfer past a permeable plate. The MHD convective heat transfer flow over vertical plate in the presence of thermophoresis and thermal conductivity were discussed by Animasaum (2015). Animasaum et al. (2016) studied the MHD heat and mass transfer flow on viscoelastic fluid over a stretching surface with magnetic field. The comparative study of heat and mass transfer flow past a stretching surface with nonlinear thermal radiation were investigated by researcher $(2015,2016,2016,2017)$. The steady MHD mixed convection flow past a horizontal stretching sheet with thermal conductivity was analysed by Animasaun (2017) and concluded that temperature profile depreciate with enhancing the melting parameter. The numerical study of MHD heat transfer flow towards a stretching surface with nonlinear thermal radiation was explained by Sathish Kumar $(2017,2017)$.On the nonlinear thermal radiation in the flow of $47 \mathrm{~nm}$ alumina-water nanofluid upper horizontal surface of paraboloid of revolution in the presence of quartic autocatalysis chemical reaction was investigated by Animasaun (2016). Abegunrin et al. (2016) discussed the effectof nonlinear thermal radiation of the flow of two non-Newtonian fluids over an upper horizontal surface of paraboloid of revolution: Boundary layer analysis. On the nonlinear thermal radiation in the flow of a nanofluid in the presence of random movement of microscopic nanoparticles waw discussed by Makinde and Animasaun (2016). 
In the present study, the flow and heat transfer characteristic of the laminar wall jet flow of a MHD nanofluid in the presence of the effect of magnetic field, thermal radiation and Biotnmber is investigated. Numerical results are determined by employing Runge-Kutta based shooting techniques. Graphs are exhibited and explained for different parameters of interest. The effect of apropos parameter on local Nusselt number, momentum and heat transfer is talked with the help table and graphs.

\section{MATHEMATICAL FORMULATION}

We consider two dimensional fully developed incompressible laminar wall jet flow of a Casson fluid at temperature Tfthrough a vertical thin slit with convective flow over a plate at temperature $\mathrm{T}_{\mathrm{w}}$ parallel to $\mathrm{x}$-axis. The spread of fluid over the plate forms a boundary layer and at far field the temperature is represented by $T_{\infty}$. A transversely applied variable magnetic field $B(x)$ is also present as shown in a Fig.1

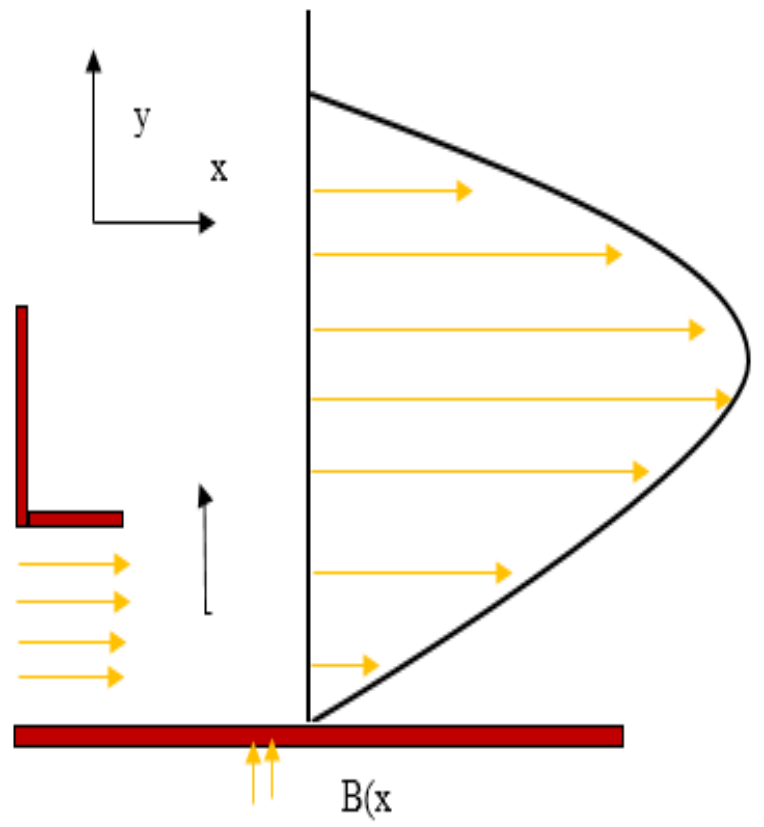

Fig. 1 Physical geometry of the problem

The equation governing the flow under the aforesaid assumption can be written as:

$$
\begin{aligned}
& \frac{\partial u}{\partial x}+\frac{\partial v}{\partial y}=0, \\
& u \frac{\partial u}{\partial x}+v \frac{\partial u}{\partial y}=-\frac{1}{\rho_{f}} \frac{\partial p}{\partial x}+v_{f}\left(\frac{\partial^{2} u}{\partial x^{2}}+\frac{\partial^{2} u}{\partial y^{2}}\right)+\frac{\sigma B^{2}(x)}{\rho_{f}} u, \\
& u \frac{\partial v}{\partial x}+v \frac{\partial v}{\partial y}=-\frac{1}{\rho_{f}} \frac{\partial p}{\partial y}+v_{f}\left(\frac{\partial^{2} v}{\partial x^{2}}+\frac{\partial^{2} v}{\partial y^{2}}\right), \\
& u \frac{\partial T}{\partial x}+v \frac{\partial T}{\partial y}=\alpha_{f}\left(\frac{\partial^{2} T}{\partial x^{2}}+\frac{\partial^{2} T}{\partial y^{2}}\right)+\frac{16 \sigma^{*}}{3 k\left(\rho c_{p}\right)_{f}} \frac{\partial}{\partial y}\left(T^{3} \frac{\partial T}{\partial y}\right),
\end{aligned}
$$

with boundary conditions

$$
\begin{aligned}
& u=0, v=0,-k_{f}=\frac{\partial T}{\partial y}=h_{f}(x)\left(T_{f}-T\right) \quad \text { at } y=0, \\
& u \rightarrow 0, v \rightarrow 0, T \rightarrow T_{\infty}, \quad y \rightarrow \infty,
\end{aligned}
$$

To get inside analysis of the problem, we use following similarity transformation.

$$
\begin{gathered}
\eta=\frac{y}{v^{1 / 2} x^{3 / 4}}, \psi=4 \sqrt{v} x^{1 / 4} f(\eta), \\
T=T_{\infty}\left(1+\left(\theta_{w}-1\right) \theta\right)
\end{gathered}
$$

We define stream function $\psi$ as

$u=\frac{\partial \psi}{\partial y}, v=-\frac{\partial \psi}{\partial x}$.

By applying eq. (6), the Eq. (1) is identically satisfied and a system of differential equations is obtained from (2)-(4) as follows.

$$
\begin{aligned}
& f^{\prime \prime}+f f f^{\prime \prime}+2 f^{\prime 2}-M f^{\prime}=0 \\
& \theta^{\prime \prime}+\operatorname{Pr} f \theta^{\prime}+\left(1+\left(\theta_{w}-1\right) \theta\right)^{3} \theta^{\prime \prime} \\
& +3 R\left(1+\left(\theta_{w}-1\right) \theta\right)^{2}\left(\theta_{w}-1\right) \theta^{\prime 2}=0
\end{aligned}
$$

We get the reduced boundary condition by utilizing Eq. (6),

$$
\begin{aligned}
& f(0)=0, f^{\prime}(0)=0, \theta^{\prime}(0)=-B i(1-\theta(0)), \\
& f(\infty) \rightarrow 1, \theta(\infty) \rightarrow 0
\end{aligned}
$$

Where, the primes denotes the differentiation with respect to $\boldsymbol{\eta}$, and $B i$ represent the reduced heat transfer parameter (Biot number).

$M=\frac{\sigma B_{0}^{2}}{\rho_{f}}, \operatorname{Pr}=\frac{v}{\alpha_{f}}, B i=\frac{h L_{c}}{k_{b}}$ Characterized by the Hartman number, Prandtl number and Biot number.

The quantities of engineering interest are friction factor and local Nusselt number $N u_{x}$ are given by $\mathrm{Re}_{x}^{\frac{1}{2}} c_{f}=f^{\prime \prime}(0)$

$$
\begin{aligned}
& N u_{x}=\frac{x q_{w}}{k\left(T_{w}-T_{\infty}\right)}, \quad q_{w}=-\left.k\left(\frac{\partial T}{\partial y}\right)\right|_{y=0} \\
& \operatorname{Re}_{x}^{-\frac{1}{2}} N u_{x}=-\theta(0),
\end{aligned}
$$

Where,

$\operatorname{Re}_{x}=\frac{u_{w}^{1 / 2} x^{1 / 2}}{v}$.

\section{RESULTS AND DISSCUSION}

The set of ODE's Eqs. (7) and (8), with the boundary condition Eq. 9 are numerically solved by using Runge- Kutta with shooting technique. The effect of various pertinent parameters viz., Magnetic field parameter, temperature ratio parameter, Biot number, radiation parameter and Prandtl number on the flow and heat transfer. Figs. 2-3 shows the graphical representation on temperature and velocity distribution for various values of the magnetic field parameter. Raising values of magnetic field parameter enhances the temperature profiles but the velocity profile showing the mixed behaviour. Generally, introducing the magnetic field create a drag force due to the Lorentz force and hence the result retarding the velocity field.

The influence of temperature ratio parameter on temperature profile is displayed in Fig. 4. It can be observed that enhancement in temperature profile when there is an enhances in the temperature ratio parameters. Physically, raising value of temperature ratio parameter to give additional heat energy to the flow filed. The effect of radiation parameter 
temperature profile is display in Fig. 5. It is noticed that increasing values of radiation parameter enhance the temperature profiles. It is due that raising value of radiation parameter release more heat energy to the flow fluid that shows an increasing behaviour of temperature profile. Fig. 6 reveal the domination Biot number on temperature fields. This is due that Biot number is the convective parameter for that it enhances the heat close to the surface.

Fig. 7 expose the nature of temperature field for various values of Prandtl number. The thermal diffusivity is conversely corresponding to Prandtlnumber, the ability of exchange the heat will be additionally limited. So we observed a fall in temperature field.Table 1 display validation for the local Nusselt number. It is noticed from the table, raising values of magnetic field parameter depreciate the heat transfer rates. Nusselt number increases for higher values of radiation parameter.

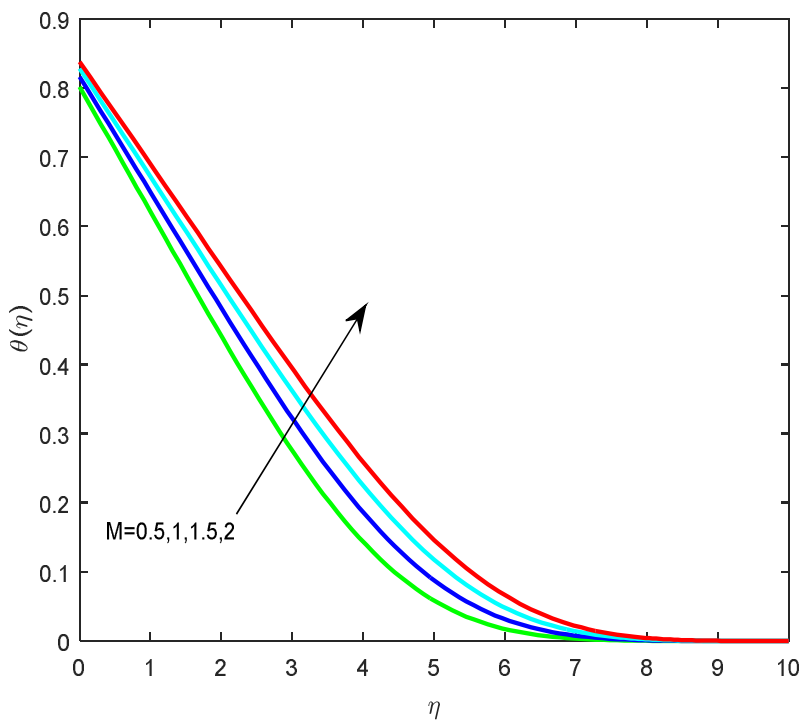

Fig. 2 Effects of $M$ on temperature profile

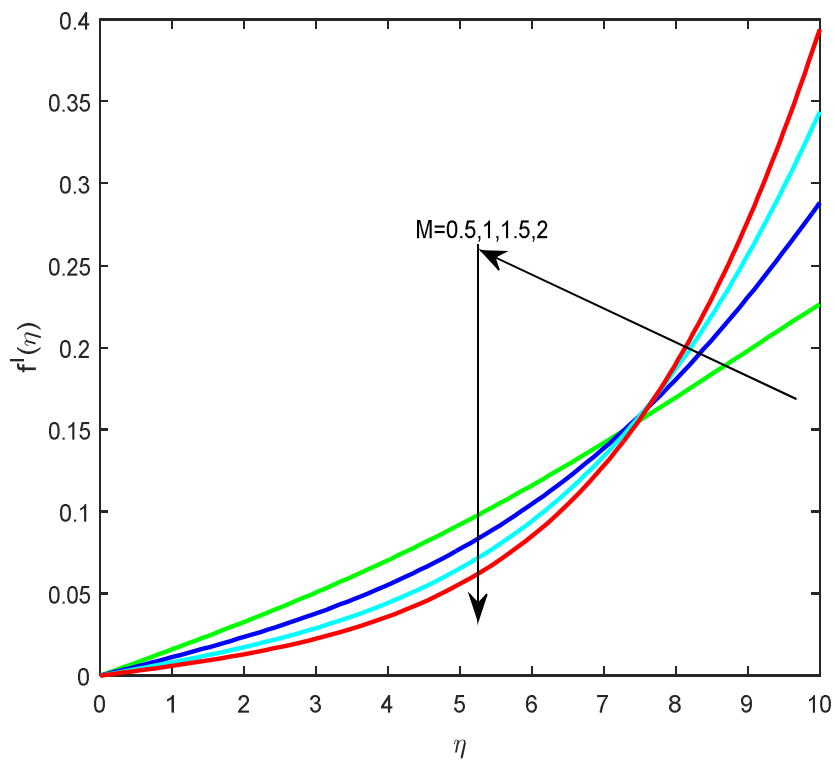

Fig. 3 Effects of $M$ on velocity profile

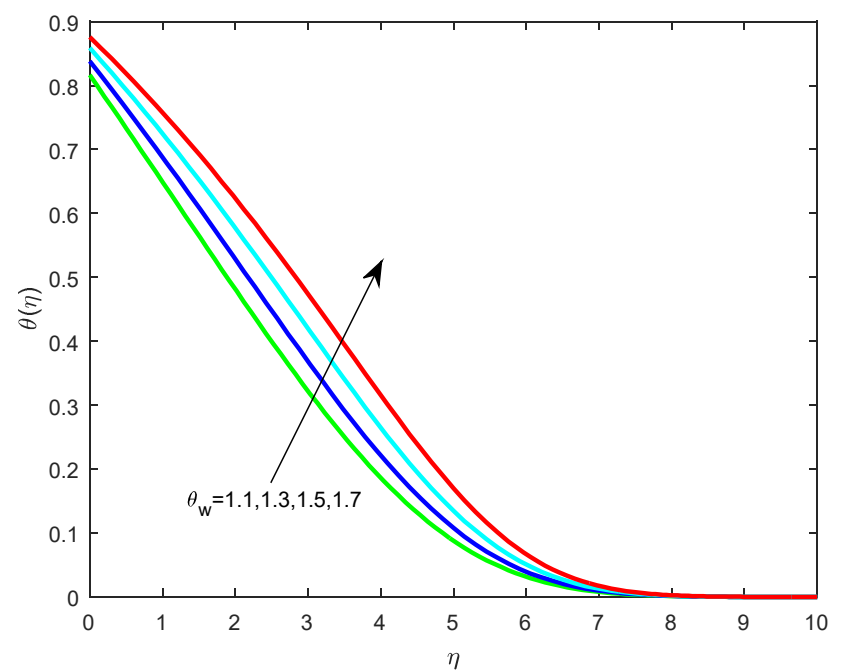

Fig. 4 Effects of $\theta_{w}$ on temperature profile

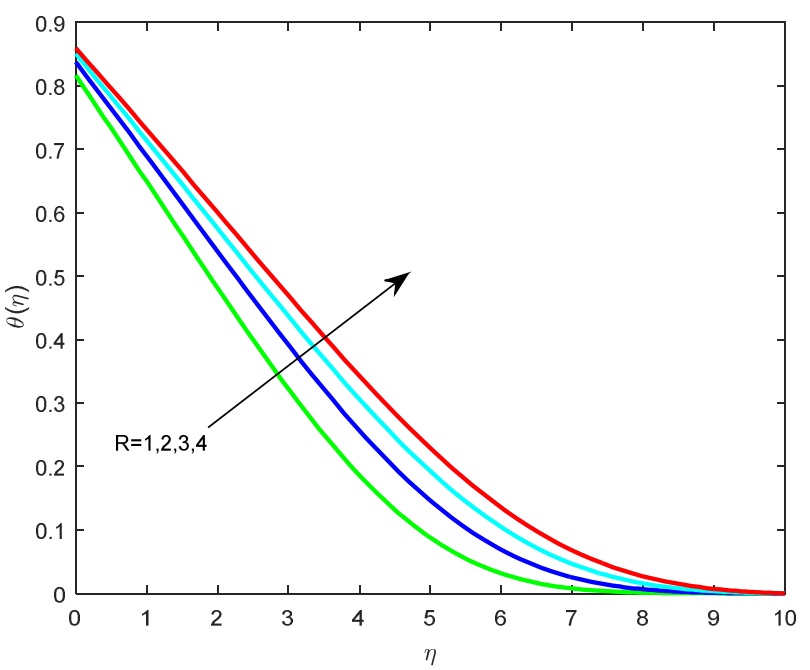

Fig. 5 Effects of $R$ on temperature profile

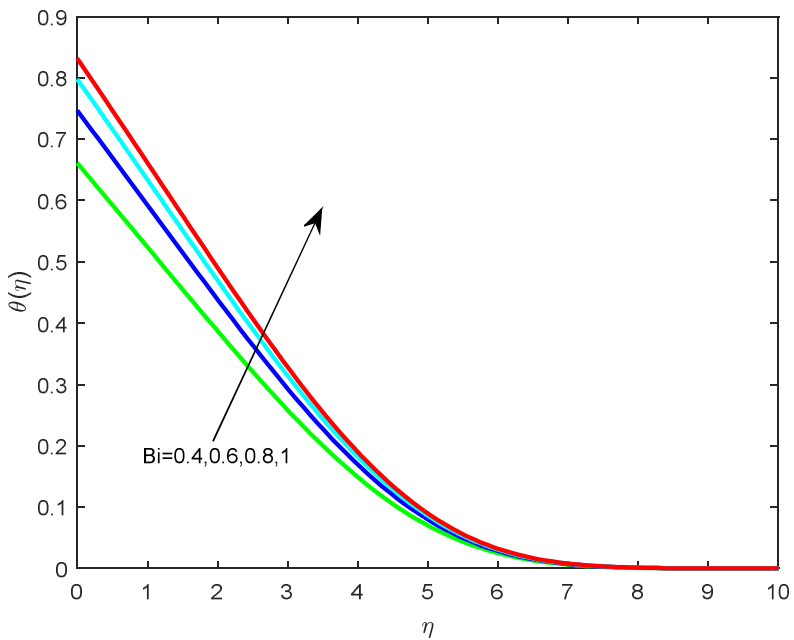

Fig. 6 Effects of $B i$ on temperature profile 


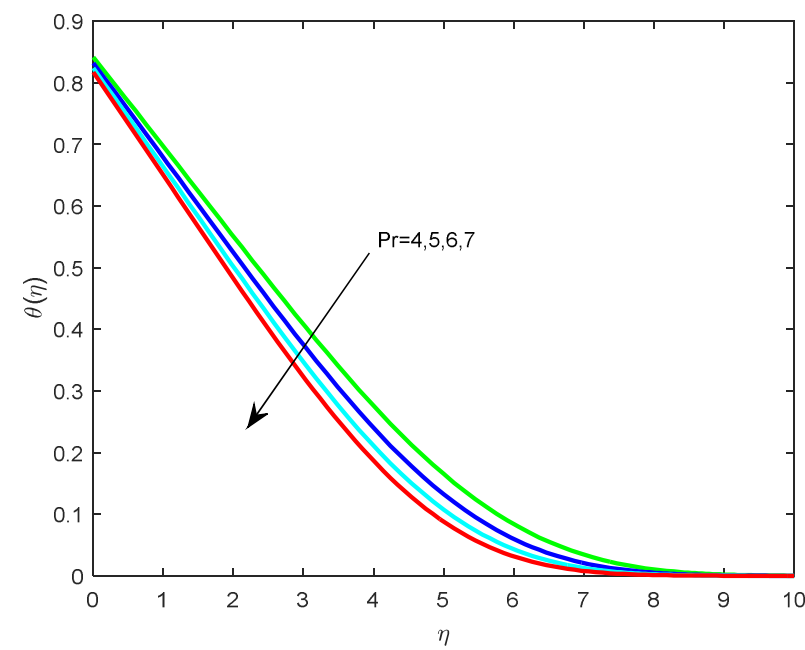

Fig. 7 Effects of P r on temperature profiles

Table 1 Physical parameter values of $-\theta^{\prime}(0)$ for general fluid

\begin{tabular}{|c|c|c|c|c|c|}
\hline$M$ & $\theta_{w}$ & $R$ & $B i$ & $\operatorname{Pr}$ & $-\theta^{\prime}(0)$ \\
\hline 0.1 & & & & & 0.178392 \\
\hline 1 & & & & & 0.165191 \\
\hline 1.5 & & & & & 0.154597 \\
\hline 2 & & & & & 0.146027 \\
\hline & 1.1 & & & & 0.165191 \\
\hline & 1.3 & & & & 0.145323 \\
\hline & 1.5 & & & & 0.127101 \\
\hline & 1.7 & & & & 0.111325 \\
\hline & & 1 & & & 0.165191 \\
\hline & & 2 & & & 0.146079 \\
\hline & & 3 & & & 0.134407 \\
\hline & & 4 & & & 0.126319 \\
\hline & & & 0.4 & & 0.135624 \\
\hline & & & 0.6 & & 0.152022 \\
\hline & & & 0.8 & & 0.161703 \\
\hline & & & 1 & & 0.168084 \\
\hline & & & & 4 & 0.143114 \\
\hline & & & & 5 & 0.151534 \\
\hline & & & & 6 & 0.158793 \\
\hline & & & & 7 & 0.165191 \\
\hline
\end{tabular}

\section{CONCLUSION}

This study presents the flow and heat transfer characteristic of the laminar wall jet flow of a MHD flow due to plate by considering magnetic field with thermal radiation and Biot number. Numerical results are determined by employing bvp5c Matlab package. Graphs are exhibited and explained for different parameters of interest. The conclusions of the present study are as follows:

- Increasing in Prandtl number enhances the Nusselt number.

- Radiation parameter have tendency to reduce localNusselt number.
- Rise in the Biot number enhances the heat transfer rate.

- An increase in temperature ratio parameter depreciate the reducedNusselt number.

- Higher value of magnetic field parameter depreciate the reduced Nusselt number

\section{NOMENCLATURE}

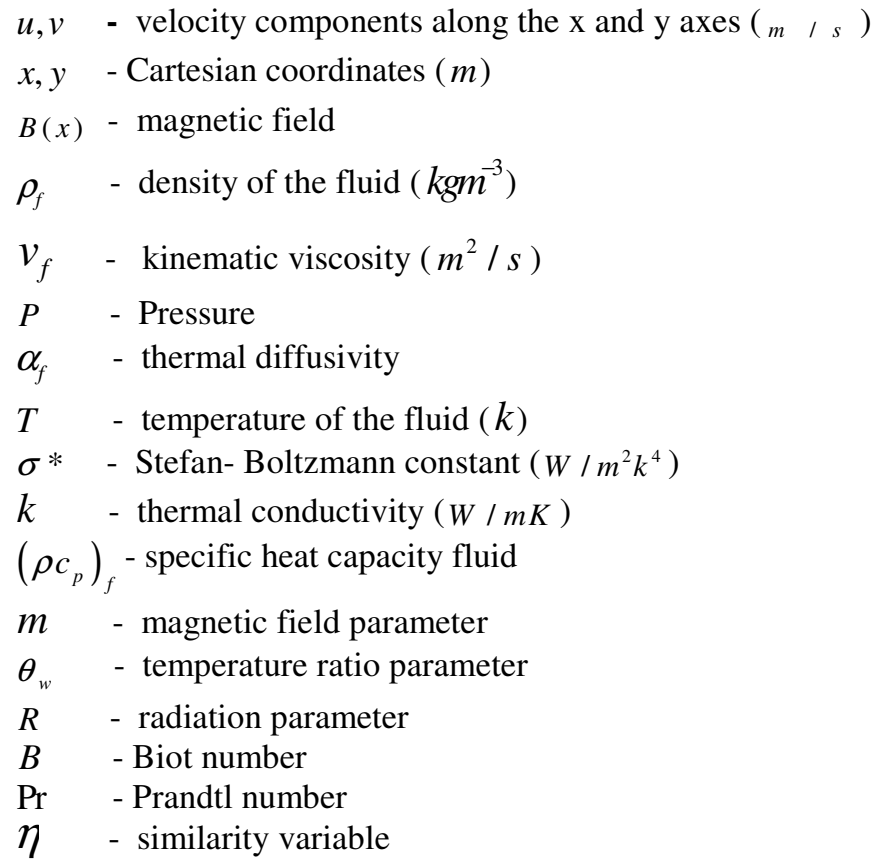

\section{REFERENCES}

Animasaum, I. L., 2015, "The Effect of Thermophoresis, Variable Viscosity and Thermal Conductivity on Free Convective Heat and Mass Transfer of Non-Darcian mhd Dissipative Casson Fluid with Suction And Nth Order of Chemical Reaction,"J. Niger. Math. Soc.,34, 11-31. http://dx.doi.org/10.1016/j.jnnms.2014.10.008

Animasaum, I. L., Raju, C. S. K. and Sandeep, N., 2016, "Unequal Diffusivities Case of Homogeneous-Hetrogeneous Reaction with in Viscoelastic Fluid Flow in the Presence of Induced Magnetic-Field and Nonlinear Thermal Radiation,"Alex. Eng. J.,55, 1595-1606. http://dx.doi.org/10.1016/j.aej.2016.01.018

Animasaun, I. L., 2017, "Melting Heat and Mass Transfer in Stagnation Point Micropolar Fluid Flow of Temperature Dependent Fluid Viscosity and Thermal Conductivity at Constant Vortex Viscosity," J. of the Egy. Math. Soc.,25, 79-85.

http://dx.doi.org/10.1016/j.joems.2016.06.007

Animasaun, I. L., 2016, "47nm Alumina-water Nanofluid flow within Boundary Layer Formed on Upper Horizontal Surface of Paraboloid of Revolution in the Presence of Quartic Autocatalysis Chemical Reaction," Alexandria Engineering J., 55(3), 2375-2389. https://doi.org/10.1016/j.aej.2016.04.030

Abegunrin, O. A., Okhuevbie, S. O. and Animasaun, I. L., 2016, "Comparision Between the Flow of Two Non-Newtonian Fluids over an Upper Horizontal Surface of Paraboloid of Revolution:Boundary Layer Analysis,"Alexandria Eng. J.,55(3), 1915-1929. https://doi.org/10.1016/j.aej.2016.08.002 
Bakier, A.Y., 2001, "Thermal Radiation Effect on Mixed Convection from Vertical Surface in Saturated Porous Media," Int. Comm. Heat Mass Transfer,28(1), 119-126.

http://dx.doi.org/10.1016/S0735-1933(01)00219-6

Hayat, T., Qayyum, S., Imtiaz, M. and Alsaedi, A., 2016, "Comparative Study of Silver and Copper Water Nanofluids with Mixed Convection and Nonlinear Thermal Radiation,"Int. J. Heat Mass Transfer, 374, 723732.

http://dx.doi.org/10.1016/j.ijheatmasstransfer.2016.06.059

Kousar, N. and Liao, S., 2011, "Unsteady Non-Similarity Boundary Layer Flows Caused by an Impulsively Stretching Flat Sheet," Nonlinear Anal. RWA, 12, 333-342.

http://dx.doi.org/10.1016/j.nonrwa.2010.06.019

Kandasamy, R., Dharmalingam, R. and SivagnanaPrabhu, K. K., 2017, "Thermal and Solutal Stratification on MHD Nanofluid Flow over a Porous Vertical Plate,"Alex. Eng. J., In press.. http://dx.doi.org/10.1016/j.aej.2016.02.029

Mahapatra, T. R. and Gupta, A. S., 2002, "Heat Transfer in Stagnation Point Flow Towards a Stretching Sheet," Heat and Mass transfer, 38, 517-521.

http://dx.doi.org/10.1007/s002310100215

Mahapatra, T.R. and Gupta, A. S., 2004 "Stagnation-Point of a Viscoelastic Fluid Towards a Stretching Surface," Int. J. Non-Linear Mech., 39, 811-820. http://dx.doi.org/10.1016/S0020-7462(03)00044-1

Mahapatra, T. R., Pal, D. andMondal, S., (2012) " Combined Effect of Thermal Radiation and Heat Generation on Natural Convection in a Square Cavity Filled with Darcy- Forchheimer Porous Medium," Int. J. of Appl. Maths. And Com., 4(4), 359-368.ISSN: 0974 - 4665.

Malvandi, A., 2016, "Film Boiling of Magnetic Nanofluids over a Vertical Plate in Presence of a Uniform Variable-Directional Magnetic Field,"J. of Mag. and Magn. Mat.,406, 95-102.

http://dx.doi.org/10.1016/j.jmmm.2016.01.008

Makinde, OO. D. and Animasaun, I. L., 2016, "Thermophoresis and Brownian motion Effects on MHD Bioconvection of Nanofluid with Nonlinear Thermal Radiation and Quartic Chemical Reaction Past an Upper Horizontal Surface of a Paraboloid of Revolution," J. of Molecular Liquids, 221, 733-743.

https://doi.org/10.1016/j.molliq.2016.06.047

Motsumi, T.G. and Makinde, O. D., 2012, "Effect of Thermal Radiation and Viscous Dissipation on Boundary Layer Flow of Nanofluids over a Permeable Moving Flat Plate," Phys. Ser. 86(4), 045003 (8pp). http://dx.doi.org/10.1088/0031-8949/86/04/045003

Pal, D., 2013, "Hall Current and MHD Effects on Heat Transfer over an Unsteady Stretching Permeable Surface with Thermal Radiation,"Comput.Math. App.,66, 1161-80. http://dx.doi.org/10.1016/j.camwa.2013.07.010

Rashidi, M.M., Ali, M., Freidoonimehr, N., Rostami, B. and Hossain, M. A., 2014, "Mixed Convection Heat Transfer for Mhd Viscoelastic Fluid Flow over a Porous Wedge with Thermal Radiation,"Adv. Mech. Eng., 2014, 735939.

http://dx.doi.org/10.1155/2014/735939
Sakiadas, B.C., 1961, "Boundary Layer Behaviour on Continuous Solid Surface, II, the Boundary Layer on a Continuous Flat Surface," AIChEJ,7, 221-225.

http://dx.doi.org/10.1002/aic.690070108

Sathish Kumar, M., Sandeep, N. and Rushi Kumar, B., 2016 “ Dual Solution form Heat and Mass Transfer in MHD Bio Convective Flow over a Stretching/ Shrinking Sheet with Suction/Injection,” Int. J. of Eng. Res. in Africa, 21, 84-101. http://dx.doi.org/10.4028/www.scientific.net/JERA.21.84

Sathis Kumar, M., Sandeep, N. and Rushi Kumar, B., 2017, "Effect of Cattano-Chritov Heat Flux on Non-linear Radiative MHD Flow of Casson Fluid Induced by a Semi-Infinite Stretching Surface,"Frontiers in H. and M. Tran.,8.

http://dx.doi.org/10.5098/hmt.8.8

Sathis Kumar, M., Sandeep, N. and Rushi Kumar, B., 2017, "Free Convective Heat Transfer of MHD Dissipative Carreau Nanofluid Flow over a Stretching Sheet,"Frontiers in H. and M. Tran., 8.

http://dx.doi.org/10.5098/hmt.8.13

Sandeep, N., Sugunamma, V. and Mohankrishna, P., 2014, "Alined Magnetic Field, Radiation and Rotation Effects on Unsteady Hydrodynamic Free Convection Flow Past an Impulsively Moving Vertical Plate in a Porous Medium," Int. J. of Eng. Maths.,2014, Id 565162.

http://dx.doi.org/10.1155/2014/565162

Sheikholeslami, M. and Ganji, D.D., 2016 "Nanofluid Convective Heat Transfer Using Semi Analytical and Numerical Approach,"J. Taiwan Inst. Chem. Eng.,65, 43-77. http://dx.doi.org/10.1016/j.jtice.2016.05.014

Sheikholeslami, M. and Ganji, D.D., 2015, "Entropy Generation of Nanofluid in Presence of Magnetic Field Using Lattice Boltzmann Method,"Phys.A,417, 273-86.

http://dx.doi.org/10.1016/j.physa.2014.09.053

Sheikholeslami, M. and Ganji, D.D., 2014, "Ferrohydrodynamics and Magnetohydrodynamic Effects on Ferrofluid Flow and Convective Heat Transfer,"Energy, 75, 400-10. http://dx.doi.org/10.1016/j.energy.2014.07.089

Sheikholeslami, M., Ganji, D.D. and Rashidi, M. M., 2016, "Magnetic Field Effect on Unsteady Nanofluid Flow and Heat Transfer Using Buongiorno Model," J. Mag. Magn. Mater, 16, 164-73. http://dx.doi.org/10.1016/i.jmmm.2016.05.026

Shaw, S., Mahanta, G. and Sibanda, P., 2016, "Non-linear Thermal Radiation Convection in a Casson Fluid Flow over a Horizontal Plate with Convective Boundary Condition,”Alex. Eng. J.,55, 1295-1304. http://dx.doi.org/10.1016/j.aej.2016.04.020

Sheikholeslami, M., Rashidi, M. M., Hayat, T. and Ganji, D.D., 2016 "Free Convection of Magnetic Nanofluid Considering MHD Viscosity Effect,"J. Mole.Liq., 218, 393-9.

http://dx.doi.org/10.1016/j.molliq.2016.02.093

Sheikholeslami, M., Ganji, D.D., YounusJavedb, M. and Ellahil, R., 2015, "Effect of Thermal Radiation on Magnetohydrodynamics Nanofluid Flow and Heat Transfer by Means of Two Phase Model," $J$. Mag. Magn. Mater,374, 36-43.

http://dx.doi.org/10.1016/i.jmmm.2014.08.021 
Sulochan, C., Kishore Kumar, M.K. and Sandeep, N., 2015, "Radiation and Chemical Reaction Effects on Mhd Thermosolutal Nanofluid Flow over a Vertical Plate in Porous Medium,"Che.and Proc. Eng. Res.,34, 28-37. ISSN 2224-7467.

Raju, C. S. K, Sandeep, N., Sulochana, C. and Sugunamma, V., 2015, "Effect of Aligned Magnetic Field and Radiation on the Flow of Ferrofluids over a Flat Plate with Non-uniform Heat Source/sink, Int. J. of Sci. and Eng.,8(2), 151-158.

http://dx.doi.org/10.12777/ijse.8.2.151-158
Raju, C. S. K. and Sandeep, N., 2016 "Heat and Mass Transfer in MHD Non-Newtonian Bio-convection Flow over a Rotaing Cone/plate with Cross Diffusion,"J. Mol. Liq.,215, 115-126.

http://dx.doi.org/10.1016/j.molliq.2015.12.058

Vajravelu, K., Prasad, K. V. and Chiu-On Ng, 2013, "Unsteady Convective Boundary Layer Flow of a Viscous Fluid at a Vertical Surface with Variable Fluid Properties,"Nonlinear Ana. Real World Alp.,14, 455- 464.

http://dx.doi.org/10.1016/j.nonrwa.2012.07.008 